\title{
Thymic masses: A radiological review
}

\author{
M K Mittal, MD, FICR; B Sureka, MD, DNB; M Sinha, MD; A Mittal, MD, DNB; B B Thukral, MD, FICR
}

Department of Radiodiagnosis and Imaging, Vardhman Mahavir Medical College and Safdarjung Hospital, New Delhi, India

Corresponding author: B Sureka (binitsurekapgi@gmail.com)

\begin{abstract}
Various thymic masses and their normal variations have different pathological and management consequences. Radiologists and clinicians should
\end{abstract} be aware of these entities so that appropriate and timely treatment can be given.

S Afr J Rad 2013;17(3):108-111. DOI:10.7196/SAJR.874

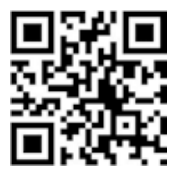

The thymus is a lymphatic organ that plays a vital role in the development and maturation of the immune system during childhood. It is bilobal, each lobe having a separate fibrous capsule that is connected to the inferior lobes of the thyroid gland by the thyrothymic ligament.

Despite the advances made in imaging technology, errors tend to occur when reporting thymic pathologies that may result in unnecessary biopsy, surgery and radiation.

The thymus is located in the anterior mediastinum. It overlies the pericardium, aortic arch, left innominate vein, and trachea. Differentiating normal from hyperplastic thymus can be difficult. On imaging, normal thymus shows the following features: absence of rounded soft-tissue masses $>7 \mathrm{~mm}$; absence of a convex contour/soft-tissue lobulation of the thymus after age 19 years and normal thymic thickness (should be $<18 \mathrm{~mm}$ before 20 years of age and $<13 \mathrm{~mm}$ after 20 years of age). ${ }^{[1]}$ Linear correlation between increased fatty infiltration of the thymus on computed tomography (CT) and decreased 18F-FDG uptake on positron emission tomography-computed tomography (PET-CT) is seen with increasing age. ${ }^{[2]}$ Various disorders can affect the thymus, e.g. epithelial, lymphoid and germ cell neoplasms; thymic cysts; hyperplasia; and hamartomatous lesions. On chest X-ray, thymic abnormalities typically manifest as focal or diffuse thickening of the anterior junction line and obliteration of retrosternal clear space. ${ }^{[3]} \mathrm{CT}$ is the primary imaging technique for evaluating suspected thymic abnormalities seen on chest radiographs as well as for detecting occult thymic masses.

\section{Rebound thymic hyperplasia}

Histologically, thymic hyperplasia can be divided into two distinct types: true hyperplasia and lymphoid (follicular) hyperplasia. The thymus commonly involutes during periods of stress. True thymic hyperplasia is diagnosed when there is $>50 \%$ increase in thymic volume over baseline, usually within a year of the stress period, with preserved microscopic features (Fig. 1). ${ }^{[4]}$ Lymphoid hyperplasia typically shows diffuse enlargement with a fine mixture of fat and lymphoid tissue, a smooth contour, and normal vessels; this in contrast to thymic tumour that is associated with a nodular contour and frequently contains necrotic or calcific foci. ${ }^{[5]}$ Thymic lymphoid hyperplasia is commonly associated with myasthenia gravis, thyrotoxicosis, Addison disease, acromegaly, thyroid cancer, connective tissue disease and early stages of human immunodeficiency virus (HIV) infection. PET-CT shows decreased uptake with standardised uptake value (SUVmax) $\leq 3.4$ in thymic hyperplasia. ${ }^{[2]}$ Chemical-shift magnetic resonance imaging (MRI) is also helpful in differentiating thymic hyperplasia from thymic neoplasms. Non-neoplastic thymic tissue shows normal fat infiltration and manifests as loss of signal on opposed-phase images relative to in-phase images. ${ }^{[3]}$

\section{Thymic cysts}

Thymic cysts can be found in the neck and/or mediastinum. They can be congenital, degenerative, inflammatory and neoplastic. Congenital cysts are usually unilocular, arise from a persistent patent thymopharyngeal duct, and usually present as a unilocular cystic mass confined to the mediastinum or extending inferiorly within the neck, paralleling the sternocleidomastoid muscle as a dumbbell-shaped left cervicothoracic cystic mass (Fig. 2). ${ }^{[6]}$ Cysts associated with neoplasms usually have a soft-tissue component.

\section{Thymic tumours Thymoma}

Thymomas are the most common primary neoplasm of the anterior mediastinum. Rarely, they can be found in ectopic locations such as the neck, or middle or posterior mediastinum. Thymomas are classified into noninvasive (benign) or invasive (malignant), and are usually seen in adults in the 5th - 6th decade but rarely in children. On imaging, noninvasive thymomas usually grow towards one side of the mediastinum, and are usually homogenous, with mild contrast enhancement (Fig. 3). Findings indicative of invasive thymoma are: encasement of mediastinal structures, infiltration of fat planes, and an irregular interface between the mass and lung parenchyma; pleural thickening or nodularity; or effusion, multifocal calcification and cystic change (Figs 4, 5). ${ }^{[7]}$ Associations of thymoma are red blood cell aplasia, hypogammaglobulinaemia, myasthenia gravis, connective tissue diseases, autoimmune disease or inflammatory bowel disease. Approximately one-third of patients with thymomas have myasthenia gravis, and approximately $10-15 \%$ of patients with myasthenia gravis have a thymoma. Dynamic MRI is used to differentiate thymomas from other anterior mediastinal masses. Thymomas tend to reach peak enhancement earlier than other mediastinal neoplasms. Noninvasive thymomas attain peak enhancement earlier than invasive thymomas. ${ }^{[3,8]}$ 


\section{Thymic carcinoma}

Thymic carcinomas account for about $20 \%$ of thymic epithelial tumours. The mean age of patients with thymic carcinomas is 50 years. It is difficult to distinguish thymic carcinomas from thymomas on the sole basis of imaging findings. On imaging, aggressive features such as central necrosis, vascular encasement, pleural/pericardial invasion, distant metastasis or mediastinal lymphadenopathy suggest thymic carcinoma. ${ }^{[9]}$ Features of vascular invasion are irregular contours of the contrast-filled vascular lumen and frank endoluminal soft tissue. ${ }^{[3,4]}$ Unlike thymomas, thymic carcinomas rarely cause paraneoplastic syndromes. PET-CT shows SUVmax $>7$ in cases of thymic carcinoma. ${ }^{[2]}$

\section{Thymic lymphoma}

Lymphoma is the most common cause of an anterior mediastinal mass in children and the second most common cause of an anterior mediastinal mass in adults. The average age of a patient with mediastinal lymphoma is 30 years. The thymus may be enlarged from primary involvement or secondary nodal invasion by lymphoma. The most common type is nodular sclerosing Hodgkin lymphoma. Homogeneous enlargement of the thymus in the presence of mediastinal or hilar lymphadenopathy is suggestive of lymphoma (Fig. 6). Differentiating recurrent lymphoma from thymic rebound hyperplasia is challenging. In thymic rebound hyperplasia, the thymic enlargement is usually symmetrical, and the contour is smooth and nonlobulated and conforms to the shape of neighbouring structures; while in recurrent thymic lymphoma,the patient is symptomatic, and the thymus is generally asymmetric and nodular and shows heterogeneous signal intensity on MRI. ${ }^{[10]}$ PET-CT shows increased uptake, with SUVmax $\geq 3.4$ in lymphoma. ${ }^{[2]}$

\section{Thymolipoma}

Thymolipomas are rare, benign, well-encapsulated thymic tumours that account for about $5 \%$ of thymic neoplasms. They usually are seen in young adults and are very large at presentation. On CT, thymolipomas predominantly show fat attenuation interspersed with fibrous septa and a connection with the thymic bed (Fig. 7).${ }^{[1]}$ Differentials of fat-containing mediastinal lesions are lipoma, liposarcoma, mediastinal lipomatosis, teratoma, diaphragmatic hernias, extramedullary erythropoiesis, lipoid pneumonia, lipoblastoma and cardiac liposarcomas. Demonstration of continuity of the mass with thymus clinches the diagnosis. Surgical resection is the treatment of choice.

\section{Thymic carcinoid}

Thymic carcinoids are rare, well-differentiated neuro-endocrine tumours. Patients with thymic carcinoids often present with endocrine disorders such as Cushing syndrome or multiple endocrine neoplasia types I and II. On imaging, they present similarly to invasive thymoma with or without calcification (Fig. 8). Thymic carcinoids are more aggressive than thymomas and cause more superior vena cava obstruction. ${ }^{[12]}$ Thymic carcinoids show avid uptake of $18 \mathrm{~F}-\mathrm{FDG}$ on PET-CT.

\section{Thymic germ cell tumours}

Primary extragonadal germ cell tumours (GCTs) are considered of primary mediastinal origin if there is neither a detectable gonadal primary nor retroperitoneal lymphadenopathy. They are usually seen during the 2nd to 4th decades of life. Lesions may be divided into seminomatous and nonseminomatous types. The most common benign mediastinal GCTs are mature teratomas. Malignant tumours are almost exclusively seen in males. Mature teratomas do not show elevation of tumour markers, while AFP elevation is diagnostic of a nonseminomatous GCT. On imaging, a combination of fluid, fat, calcification and soft-tissue component is highly specific for teratoma (Fig. 9). Calcification is seen in $50 \%$ and fat in 50 $75 \%$ of cases of teratoma. Malignant GCT usually shows aggressive features with the presence of solid components and absence of fat/calcification with elevation of tumour markers. Seminomatous GCT grows slowly, is usually large and homogenous, and has a good prognosis (Fig. 10). Nonseminomatous malignant GCTs are aggressive tumours thatgrow rapidly and are more heterogenous, with cystic/necrotic changes (Fig. 11) ${ }^{[9]}$

\section{Thymic sarcomas}

Thymic sarcomas are extremely rare. They have a nonspecific appearance at cross-sectional imaging with more aggressive features at initial presentation, and carry a poor prognosis.

\section{Secondary tumours of the thymus}

Secondary tumours of the thymus include invasion of the thymus by lung carcinoma and involvement of the thymus via lymphatic pathways in cancers of the head, neck, abdomen and pelvis. Langerhans cell histiocytosis also frequently involves the thymus in the form of an anterior mediastinal mass and may be visible at imaging. ${ }^{[13]}$

1. Baron RL, Lee JK, Sagel SS, Peterson RR. Computed tomography of the normal thymus. Radiology 1982;142(1):121-125.

2. Sharma P, Singhal A, Kumar A, Bal C, Malhotra A, Kumar R. Evaluation of thymic tumours with 18F-FDG PET-CT: A pictorial review. Acta Radiol 2013;54(1):14-21. [http://dx.doi.org/10.1258/ ar.2012.120536]

3. Rosado-de-Christenson ML, Strollo DC, Marom EM. Imaging of thymic epithelial neoplasms. Hematol Oncol Clin North Am 2008;22(3):409-431. [http://dx.doi.org/10.1016/j.hoc.2008.03.011]

4. Peters R, Peters O, Braak S, Verschakelen J. Pathology of the thymus on CT imaging. JBR-BTR 2012;95(5):281-288.

5. Kissin CM, Husband JE, Nicholas D, Eversman W. Benign thymic enlargement in adults after chemotherapy: CT demonstration. Radiology 1987;163(1):67-70.

6. Ibrahim M, Hammoud K, Maheshwari M, Pandya A. Congenital cystic lesions of the head and neck. Neuroimag Clin N Am 2011;21(3):621-639. [http://dx.doi.org/10.1016/j.nic.2011.05.006]

7. Nasseri F, Eftekhari F. Clinical and radiologic review of the normal and abnormal thymus: Pearls and pitfalls. RadioGraphics 2010;30(2):413-428. [http://dx.doi.org/10.1148/rg.302095131]

8. Sakai S, Murayama S, Soeda H, et al. Differential diagnosis between thymoma and non-thymoma by dynamic MR imaging. Acta Radiol 2002;43(3):262-268.

9. Webb RW. The mediastinum: mediastinal masses. In: Webb RW, Higgins C, eds. Thoracic Imaging: Pulmonary and Cardiovascular Radiology. Philadelphia, Pa: Lippincott, Williams \& Wilkins, 2005:212-270.

10. Wernecke K, Vassallo P, Rutsch F, Peters PE, Potter R. Thymic involvement in Hodgkin disease: CT and sonographic findings. Radiology 1991;181(2):375-383.

11. Molinari F, Bankier AA, Eisenberg RL. Fat-containing lesions in adult thoracic imaging. AJR 2011;197(5):W795-W813. [http://dx.doi.org/10.2214/AJR.11.6932]

12. Kogan J. Carcinoid tumour of the thymus. Postgrad Med 1984;75(1):291-296.

13. Junewick JJ, Fitzgerald NE. The thymus in Langerhans' cell histiocytosis. Pediatr Radiol 1999;29(12):904-907. 


\section{PICTORIAL ESSAY}

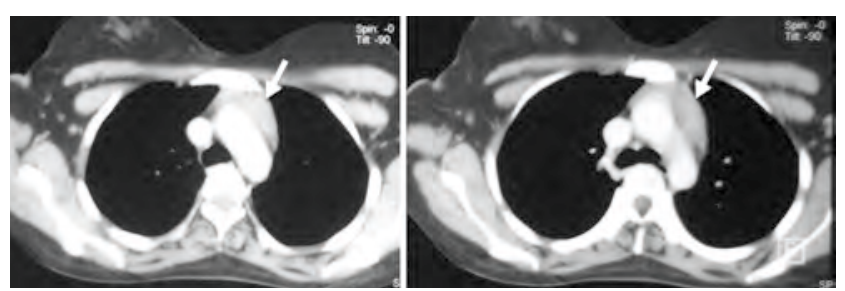

Fig.1. Computed tomography scan showing rebound thymic hyperplasia (arrows) in a 45-year-old woman post chemotherapy for cervical cancer.

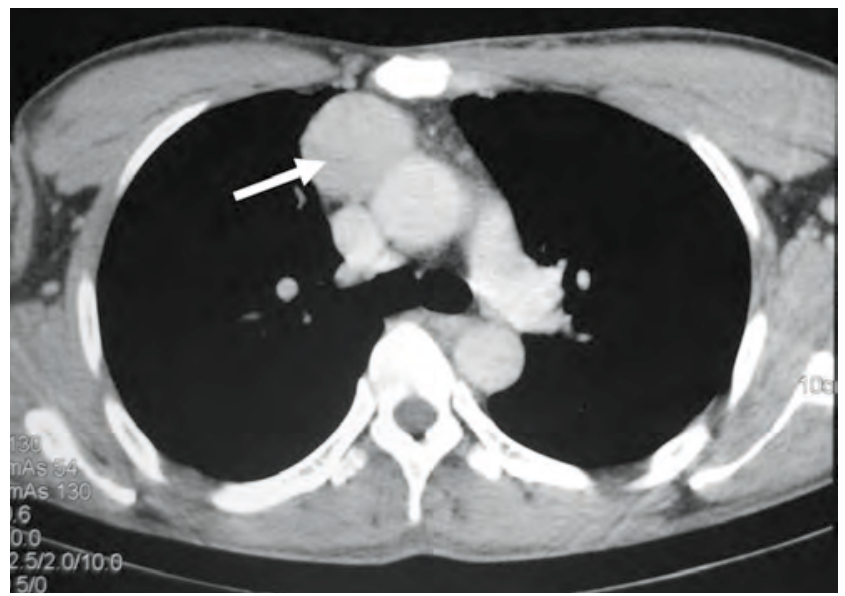

Fig. 3. Axial CT image shows homogenous $3 \mathrm{~cm}$ soft-tissue mass with mild enhancement in the anterior mediastinum in a case of noninvasive thymoma.

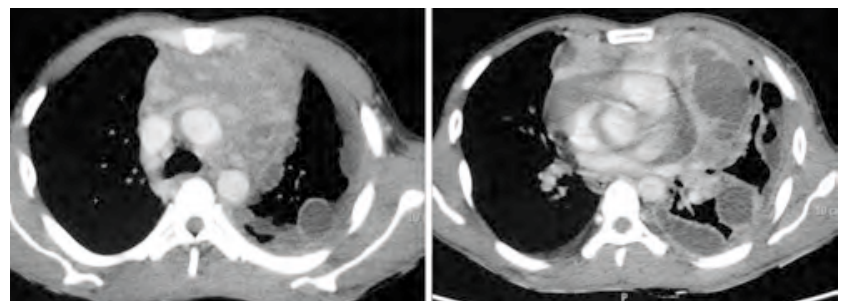

Fig. 5. Axial CT images showing a heterogenously enhancing anterior mediastinal mass with mild left pleural effusion and pleural implants in another case of invasive thymoma.

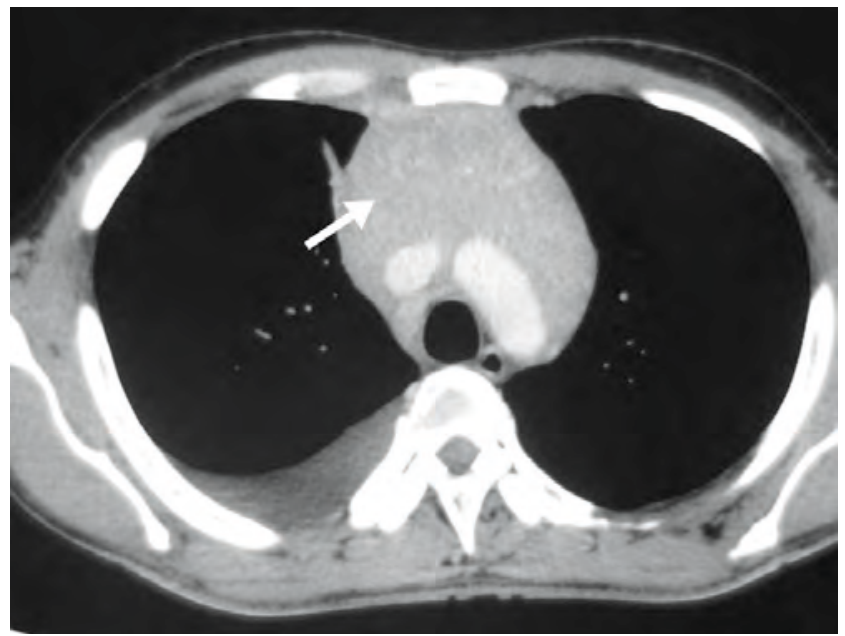

Fig. 6. Axial CT image showing homogenously enhancing soft tissue anterior mediastinal mass (arrow) replacing the thymus in a case of thymic lymphoma.

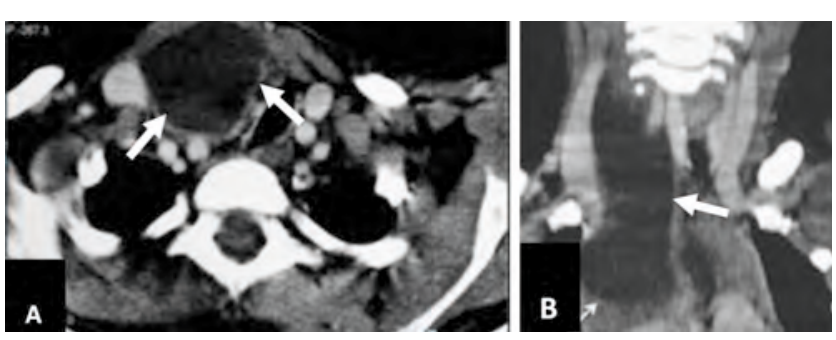

Fig. 2. Thymic cyst: (A) Axial contrast-enhanced CT scan shows a cystic lesion (arrows) in the right side of the neck caudal to the thyroid gland, displacing the trachea to the left. (B) Coronal reformatted image shows the lesion (arrow) is parallel to the sternocleidomastoid muscle and extends into the upper mediastinum.

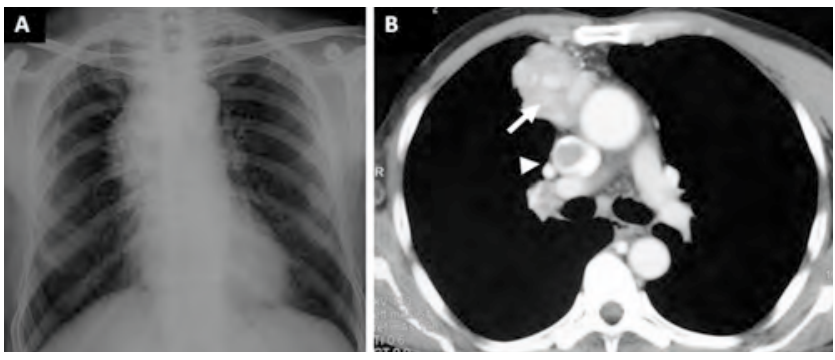

Fig. 4. (A) Chest radiograph showing an anterior mediastinal mass silhouetting the ascending aorta. (B) Axial CT image showing solid anterior mediastinal mass (arrow) with direct invasion of the superior vena cava (arrowhead) in a case of invasive thymoma.

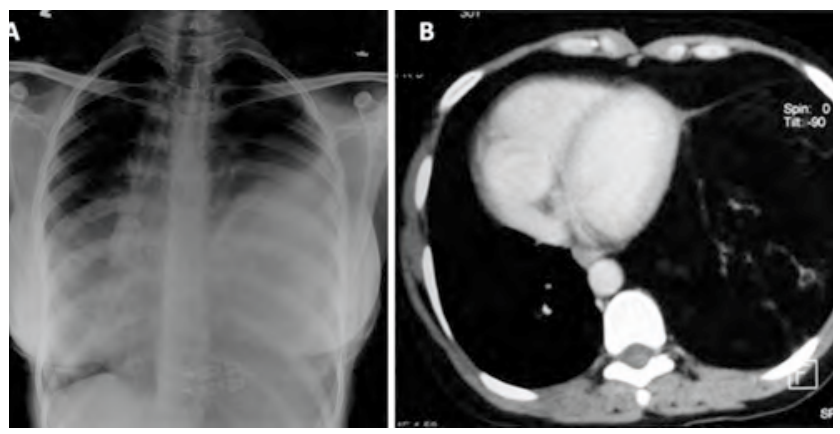

Fig. 7. (A) Chest skiagram reveals non-homogeneous opacities in bilateral mid and lower zones predominantly in central regions silhouetting the cardiac borders with blunting of bilateral costophrenic and cardiophrenic angles. (B) Axial CT image of the same patient showing a well-marginated fat attenuation mass with thin strands of fibrous septa on both sides of the hemithorax.

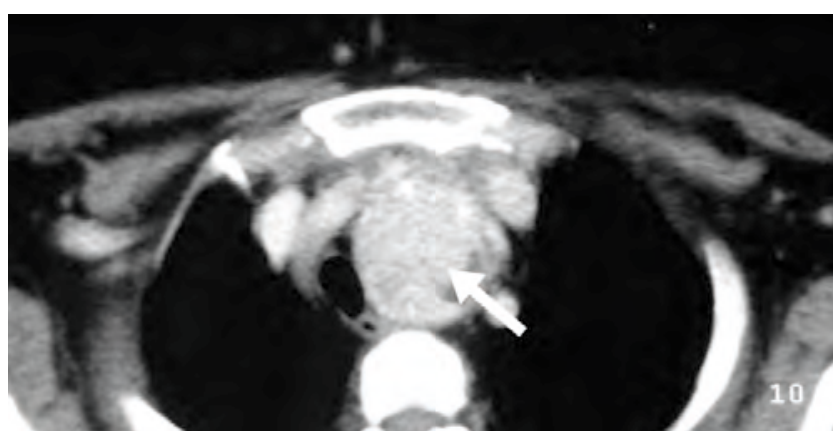

Fig. 8. Axial CT image in a patient with Cushing's syndrome showing ectopic thymic neuroendocrine tumour (arrow). 


\section{PICTORIAL ESSAY}

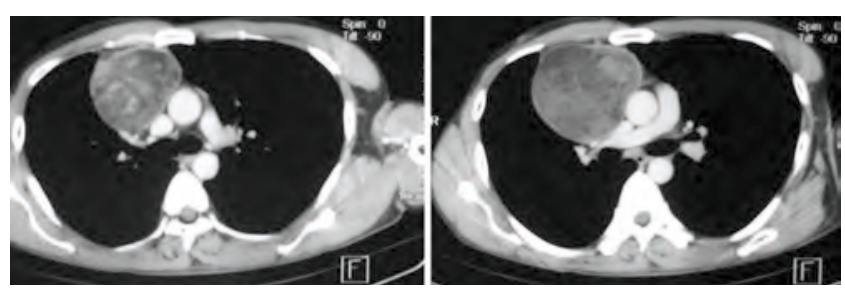

Fig. 9. Axial CT image in a 30-year-old man showing complex anterior mediastinal mass containing cystic, fat and soft-tissue components in a case of mature teratoma.

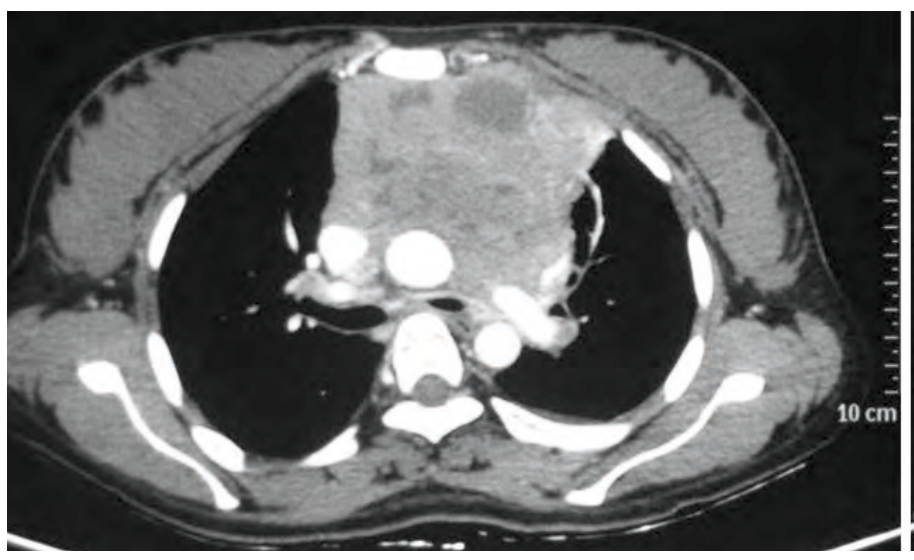

Fig. 11. Axial CT image in a 30-year-old woman showing heterogenously enhancing anterior mediastinal mass with cystic/necrotic changes in a proven case of nonseminomatous germ cell tumour.

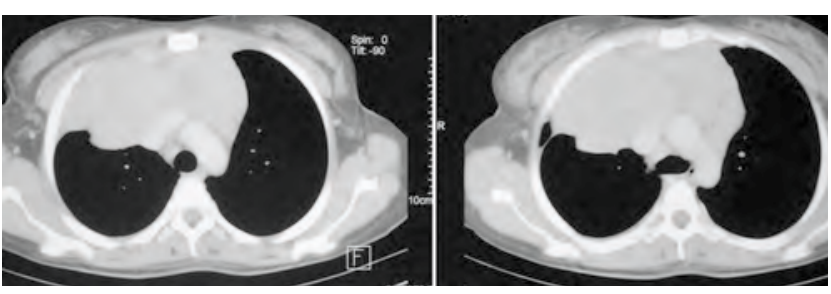

Fig. 10. Axial CT image in a 24-year-old woman showing homogenously enhancing anterior mediastinal mass in a case of seminomatous germ cell tumour.

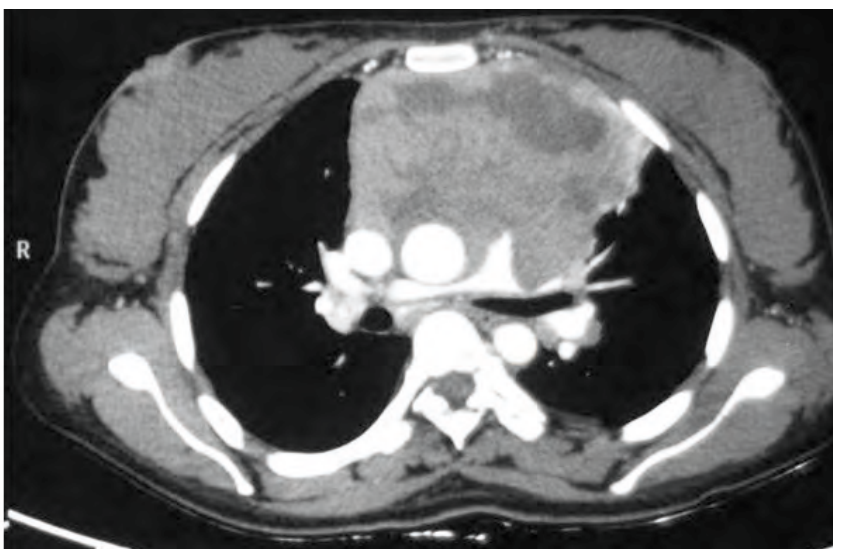

of 